\title{
Note
}

\section{Construction of a chromosome-assigned, sequence-tagged linkage map for the radish, Raphanus sativus $L$. and QTL analysis of morphological traits}

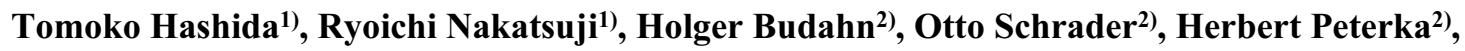 \\ Tatsuhito Fujimura ${ }^{3)}$, Nakao Kubo*1) and Masashi Hirai') \\ 1) Graduate School of Life and Environmental Sciences, Kyoto Prefectural University, Seika, Kyoto 619-0244, Japan \\ 2) Julius Kühn-Institut (JKI), Federal Research Centre for Cultivated Plants, Institute for Breeding Research on Horticultural and Fruit \\ Crops, D-06484 Quedlinburg, Germany \\ 3) Graduate School of Life and Environmental Sciences, University of Tsukuba, Tsukuba, Ibaraki 305-8572, Japan
}

\begin{abstract}
The radish displays great morphological variation but the genetic factors underlying this variability are mostly unknown. To identify quantitative trait loci (QTLs) controlling radish morphological traits, we cultivated $94 \mathrm{~F}_{4}$ and $\mathrm{F}_{5}$ recombinant inbred lines derived from a cross between the rat-tail radish and the Japanese radish cultivar 'Harufuku' inbred lines. Eight morphological traits (ovule and seed numbers per silique, plant shape, pubescence and root formation) were measured for investigation. We constructed a map composed of 322 markers with a total length of $673.6 \mathrm{cM}$. The linkage groups were assigned to the radish chromosomes using disomic rape-radish chromosome-addition lines. On the map, eight and 10 QTLs were identified in 2008 and 2009, respectively. The chromosome-linkage group correspondence, the sequence-specific markers and the QTLs detected here will provide useful information for further genetic studies and for selection during radish breeding programs.
\end{abstract}

Key Words: radish, STS, linkage map, chromosome assignment, QTL.

\section{Introduction}

The radish, Raphanus sativus L. $(2 n=18)$, is an important vegetable, especially in Asia (see Kaneko et al. 2007 for a review), and is also used as fodder and oil plants. Its hypertrophied root with hypocotyl is mainly used for human consumption. Young pods are also eaten as a vegetable in tropical Asia. Although nomenclature is complex, cultivated radishes are classified into three main types based on edible parts: fodder and oilseed radish (convar. oleifer Alef.), seedpod or rat-tailed radish (convar. caudatus (L.f.) Pistr.) and small- and large-rooted radish (convar. sativus L.) (Crisp 1995). Wide morphological variations are observed in the roots of local and commercial radish cultivars. Despite such importance, few studies have been undertaken to analyze the genetic factors that determine the variability of agronomic traits in radish. The radish genome is closely related to the Brassica genomes carried by the vegetable and oilseed plants, Brassica rapa, B. nigra, B. oleracea and the amphidiploid species (B. juncea, B. napus and B. carinata). Linkage maps of Brassica species have been extensively studied

Communicated by H. Fukuoka

Received October 18, 2012. Accepted January 27, 2013.

*Corresponding author (e-mail: nk0103@kab.seika.kyoto.jp) and numerous genetic loci for qualitative and quantitative traits have been identified (see Schmidt and Bancroft 2011 for a review). The whole genome data are now available for B. rapa (Wang et al. 2011). In contrast, limited number of genetic maps has been constructed for radish (Bett and Lydiate 2003, Budahn et al. 2009, Kamei et al. 2010, Li et al. 2011, Nakatsuji et al. 2011, Shirasawa et al. 2011, Tsuro et al. 2005, 2008, Xu et al. 2012), few of which have included agronomic trait loci.

In addition to the molecular genetic map, cytogenetic study can provide information complementary to that from genetic and physical maps. A complete set of disomic raperadish chromosome-addition lines has also been developed (Budahn et al. 2008) and the position of a locus for resistance against the beet cyst nematode has been determined using the chromosome-addition lines (Budahn et al. 2009). However, the cytogenetic data have not yet been integrated to sequence-specific molecular markers of radish.

Molecular markers provide a powerful tool for gene mapping and efficient selection of the genotypes having desired agronomic traits. This strategy is known as markerassisted selection (MAS). Among the molecular markers, simple sequence repeats (SSRs), tandem repeats of short DNA sequences from one to six base pairs, are useful tools. Because of their high level of polymorphism, ubiquity and 
co-dominance, SSRs have become a valuable source of molecular markers in genetic analysis (Jones et al. 2009). SSRs have also been useful to detect the conserved order of genes among related genomes, referred to as "synteny". Although we have previously developed 417 SSRs, including 293 genomic SSRs, from the rat-tailed radish, Japanese cultivar 'Harufuku' and their $\mathrm{F}_{1}$ plant (Nakatsuji et al. 2011), most of our SSRs have not yet been employed for mapping studies. The rat-tail radish and 'Harufuku' have several differences in vegetative and reproductive morphology traits (e.g. ovule and seed numbers, plant shape, leaf pubescence and root formation). Such the differences between the two radishes allowed us to investigate genetic loci controlling their morphologies.

The objective of this study was the construction of a radish linkage map containing radish SSR and sequence-tagged site (STS) markers, assignment of the linkage groups (LGs) to the chromosomes and mapping of quantitative trait loci (QTLs) for vegetative and generative characters.

\section{Materials and Methods}

\section{Plant materials}

A population of $94 \mathrm{~F}_{4}$ and $\mathrm{F}_{5}$ recombinant inbred lines (RILs) was developed from a cross between the rat-tail radish and the Japanese radish 'Harufuku' inbred lines (Saya-S and Haru-S, respectively) using a single-seed-descent of the $F_{1}$ plant after four generations of selfings. The rat-tail radish is grown in Southeast Asia for its edible young seed-pods (Kitamura 1958). Instead of many seed-pods and larger plant top, the rat-tailed radish forms no fleshy root (Supplemental Fig. 1A). Its leaves are erect without pubescence on the surface. The 'Harufuku' is classified into Spring radish, which has a white, wedge-shaped root (Furusato and Miyazawa 1958) (Supplemental Fig. 1B). It has hairy, prostrate leaves. Although these two cultivars are classified as different convarieties (caudatus and sativus), they intercrossed freely. Both parental lines were supplied by the NARO Institute of Vegetable and Tea Science, Tsu, Japan. The parental lines Saya-S and Haru-S used in this study are highly homozygous by five or six successive selfings. A commercial rat-tail radish cultivar (Chia Tai Co. Ltd., Bangkok, Thailand) (Supplemental Fig. 1A) was used for field tests instead of the original parental line Saya-S because of the severe inbred depression in the Saya-S. Note that the 'Harufuku' inbred line Haru-S used in this study had shorter main root and more lateral roots than the original cultivar (Supplemental Fig. 1B), probably being an aspect of inbred depression.

\section{Field tests}

The population of $94 \mathrm{~F}_{4}$ or $\mathrm{F}_{5}$ families as well as their parental lines was used for investigation of eight agronomic traits (Table 1). For scoring the ovule $(\mathrm{OV})$ and seed numbers $(S E)$ per silique, each $\mathrm{F}_{4}$ RIL was grown in a heated greenhouse under a natural photoperiod until June 2008. For the other agronomic traits, $F_{5}$ RILs were cultivated under natural conditions in an open field of the Kyoto Prefectural University Farm (Seika, Kyoto, Japan). Six plants per each RIL were cultivated from mid-September to mid-December. The field test was performed once in 2008 and 2009, respectively. Plant shape $(P S)$ and pubescence $(P u b)$ were categorized visually on 1-5 scales two months after sowing (Table 1 and Supplemental Fig. 2). Fresh weights for the following parts were measured at harvest (three months after sowing). After measurement of whole plant weight $(W W)$, plant tops were removed to measure upper part weight $(U W)$. The remainder (hypocotyls and roots) was taken as whole root weight $(R W)$. The whole root was separated into main and lateral roots to measure main root weight $(M W)$ and lateral root weight, respectively. The lateral root weight trait was excluded from further analysis because the variation was small (data not shown). After removal of extreme outliers, the mean score of the six $\mathrm{F}_{5}$ plants for each line was used as data for each $\mathrm{F}_{4}$ line.

Genotyping, linkage mapping, synteny detection and QTL analysis

Genomic DNA was extracted using the DNeasy Plant Mini Kit (Qiagen, Valencia, CA, USA) from a bulk of leaf tissue consisting on average of $13 \mathrm{~F}_{5}$ plants for each line, because the initial DNA extracted from $\mathrm{F}_{4}$ plants by the CTAB method was not of sufficient quality for AFLP analysis. We confirmed no significant incongruence in genotypes between the $F_{4}$ and the bulked $F_{5}$ DNAs for each line with tested markers (data not shown). AFLP was performed according to method described by Tsuro et al. (2008). SSR primers from B. rapa (Suwabe et al. 2006) and radish (Nakatsuji et al. 2011, Wang et al. 2007, this study) were randomly picked up to screen polymorphic markers between the parental lines. PCR amplification and electrophoresis were carried out as described previously (Saito et al. 2006). The published STS markers for B. rapa and radish (Kamei et al. 2010, Li et al. 2011, Niikura and Matsuura 1998, Saito et al. 2006) were also used. New STS markers were developed from the sequences of Arabidopsis, B. rapa and radish at the National Center for Biotechnology Information (NCBI) (http://www.ncbi.nlm.nih.gov/nucleotide/) and the RadishDatabase (Supplemental Fig. 3, http://radish.plantbiology. msu.edu/index.php/Sequences:All) (their accession nos. AB630374-AB630382 and AB611697-AB611700).

Segregation of each marker in the bulked $\mathrm{F}_{5}$ families was scored and treated as a genotype of the $\mathrm{F}_{4}$ line. Markers deviating significantly $(P<0.001)$ from the expected segregation ratio were excluded from the analysis. A linkage map was constructed using JoinMap ver. 3.0 (Van Ooijen and Voorrips 2001) as described previously (Saito et al. 2006). To detect potential synteny, nucleotide sequences of the mapped sequence-tagged markers were searched for the Arabidopsis genome data using a BlastN algorithm under the default parameter sets at the NCBI. QTL analysis was performed using MapQTL 6 software (Van Ooijen 2009) as described previously (Kubo et al. 2010). The significant 
logarithm of odds (LOD) thresholds of each QTL $(\alpha=0.05)$ were estimated by 1000 permutations. They ranged from $2.75(\mathrm{UW}$ in 2009$)$ to $3.00(\mathrm{OV})$.

\section{Chromosomal assignment}

A complete set of disomic rape-radish chromosomeaddition lines (Budahn et al. 2008) was used for assignment of the chromosomes corresponding to the radish LGs. Each disomic addition line has one of the nine pairs of the radishderived chromosomes (pairs AA-II) on the synthetic B. napus background (Peterka et al. 2004). Thirty six markers (33 radish SSRs, one radish STS and two B. rapa SSRs) were screened for PCR, using the genomic DNAs of the nine disomic addition lines as well as their parental lines as templates. Loci which yielded radish-specific PCR products in the particular chromosome addition line (Supplemental Fig. 5, arrowheads) were assigned to one of the nine radish chromosomes (Supplemental Fig. 5, letters).

\section{Results}

\section{Analysis of phenotypic variation}

Phenotypic variation of eight agronomic traits examined in RILs and the parental lines are shown in Supplemental Fig. 4 and summarized in Table 1. The mean scores of the rat-tailed radish were larger than those of the Haru-S in $O V$, $P S, W W$ and $U W$ in each year (and presumably also in $S E$, see Discussion) (Table 1). It was vice versa in Pub, $R W$ and $M W$. The majority of RILs were roughly segregated into two categories of pubescence $(P u b)$, presence (categories 2-5) and absence (category 1), whose distribution was biased towards the presence of pubescence (Supplemental Fig. 4D). Since the segregation ratio, divided into the above two categories of $P u b$ as a qualitative trait, was significantly deviated at $P<0.0001$ from an expected segregation ratio for RIL population, we conducted the QTL analysis for $P u b$ as below. For the other traits, RILs did not show a discrete segregation, suggesting that these traits are controlled by multiple genes. The distributions of RILs for weight traits $(W W, U W$, $R W$ and $M W$ ) were shifted towards larger values in 2009 than seen in 2008 (Supplemental Fig. 4E-4H, gray and black bars, respectively) probably because of an environmental effect. In root weight traits $(R W$ and $M W)$, RILs with transgressive weight exceeding the values for the Haru-S (Supplemental Fig. 4G-4H, pink arrows) were found. In $\mathrm{OV}$, transgression beyond the value for the rat-tailed radish also occurred (Supplemental Fig. 4A, green arrow).

\section{Linkage mapping, $L G$ assignment using the disomic chro- mosome addition lines and synteny to Arabidopsis}

The map comprised nine LGs, including 163 radish AFLPs, 97 radish SSRs (75 genomic SSRs and 22 ESTSSRs), 37 radish STSs, 15 B. rapa SSRs, seven B. rapa STSs and three Arabidopsis STSs (Fig. 1). Of these, 10 SSR and 25 STS markers were newly developed or modified in this study (Table 2). The total map length was $673.6 \mathrm{cM}$, with an average marker interval of $2.1 \mathrm{cM}$. A total of 36 markers were tested for assignment of the present LGs to radish chromosomes using the disomic addition lines (Budahn et al. 2008) (Supplemental Fig. 5 for examples). Of these, 19 markers (18 radish SSRs and one radish STS) were assigned to the radish chromosomes (Fig. 1, gray boxes). There was no incongruence between the markers and the assigned chromosomes. Thus, we also refer each LG to our previous nomenclature (Chrs $\mathrm{A}-\mathrm{I})$ as well as LG numbers (R1-R9) given by Li et al. (2011) (Fig. 1, parentheses). Nucleotide sequences of 138 sequence-tagged markers showed homology to Arabidopsis (data not shown). Most of the homologies to the five Arabidopsis chromosomes were divided into many segments and dispersed throughout the present

Table 1. List of traits analyzed and the phenotypic values of the $\mathrm{F}_{4}$ or $\mathrm{F}_{5}$ population and the parental lines

\begin{tabular}{|c|c|c|c|c|c|c|}
\hline Trait & Abbreviation & Year & $\begin{array}{c}\text { Mean }( \pm \mathrm{SD}) \\
\text { in RIL }\end{array}$ & $\begin{array}{l}\text { Range } \\
\text { in RIL }\end{array}$ & $\begin{array}{c}\text { Mean }( \pm \mathrm{SD}) \\
\text { in rat-tail radish }\end{array}$ & $\begin{array}{c}\text { Mean }( \pm \mathrm{SD}) \\
\text { in Haru-S }\end{array}$ \\
\hline Ovule number per silique & $O V$ & 2008 & $9.93 \pm 2.07$ & $5.00-16.25$ & $9.40 \pm 0.80$ & $6.60 \pm 0.49$ \\
\hline Seed number per silique & $S E$ & 2008 & $3.09 \pm 1.00$ & $1.14-5.55$ & N.D. ${ }^{c}$ & $3.04 \pm 1.51$ \\
\hline \multirow[t]{2}{*}{ Plant shape } & $P S$ & 2008 & $3.1 \pm 0.8$ & $1-5^{a}$ & 5 & 1 \\
\hline & & 2009 & $3.2 \pm 0.7$ & $1-5^{a}$ & 5 & 1 \\
\hline \multirow[t]{2}{*}{ Pubescence } & $P u b$ & 2008 & $2.9 \pm 1.3$ & $1-5^{b}$ & 1 & 5 \\
\hline & & 2009 & $2.9 \pm 1.3$ & $1-5^{b}$ & 1 & 5 \\
\hline \multirow[t]{2}{*}{ Whole plant weight (g) } & $W W$ & 2008 & $598 \pm 236$ & $142.6-1516$ & $982 \pm 311$ & $429 \pm 173$ \\
\hline & & 2009 & $1267 \pm 458$ & $426.7-2890$ & $2486 \pm 1140$ & $1038 \pm 252$ \\
\hline \multirow[t]{2}{*}{ Upper part weight (g) } & $U W$ & 2008 & $416 \pm 173$ & $119.8-1142$ & $879 \pm 268$ & $232 \pm 86.9$ \\
\hline & & 2009 & $1005 \pm 385$ & $341.7-2518$ & $2346 \pm 1096$ & $716 \pm 174$ \\
\hline \multirow[t]{2}{*}{ Whole root weight (g) } & $R W$ & 2008 & $180 \pm 119$ & $16.8-648$ & $103 \pm 46.5$ & $197 \pm 88.4$ \\
\hline & & 2009 & $249 \pm 174$ & $26.0-801$ & $135 \pm 63.9$ & $320 \pm 83.4$ \\
\hline \multirow[t]{2}{*}{ Main root weight $(\mathrm{g})$} & $M W$ & 2008 & $148 \pm 96.3$ & $15.7-534$ & $87.5 \pm 43.3$ & $175 \pm 76.3$ \\
\hline & & 2009 & $239 \pm 165$ & $26.0-744$ & $123 \pm 63.3$ & $314 \pm 84.8$ \\
\hline
\end{tabular}

${ }^{a} 1$ = prostrate; 2 = semi-prostrate; 3 = intermediate; 4 = open; 5 = erect, see Supplemental Fig. 2.

${ }^{b} 1=$ no hair; $2=$ sparse; $3=$ intermediate; $4=$ abundant; $5=$ very abundant hair like Haru-S.

${ }^{c}$ N.D., not determined. 

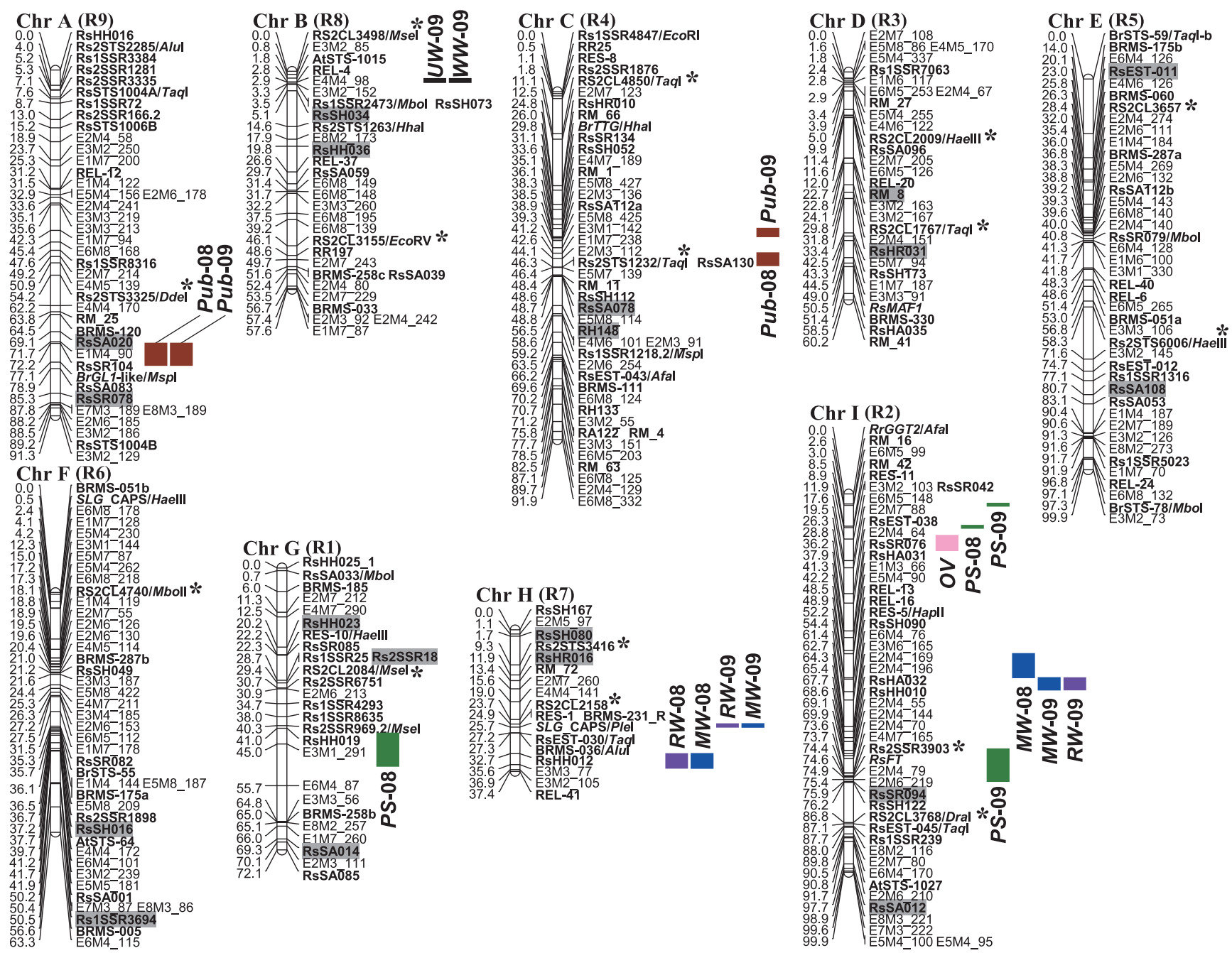

Fig. 1. A linkage map of radish and the locations of QTLs for eight agronomic traits. Linkage groups were referred to chromosome numbers (Chrs A-I) (Budahn et al. 2008) and LG numbers (R1-R9) (Li et al. 2011). Marker names and genetic distances (cM) are shown on the right and left of each LG, respectively. SSR and STS markers are indicated with bold-face type. Markers used for the assignment to radish chromosomes and LG numbers are indicated with gray boxes and asterisks, respectively. Locations of QTLs are indicated with differentially-colored, vertical thick bars, the lengths of which correspond to the map positions in Table 3. The QTLs detected in 2008 and 2009 are represented with suffix numbers such as "-08" and "-09", respectively.

radish map. Relatively large clusters were found in all radish LGs, which may represent syntenies to Arabidopsis.

\section{QTL analysis}

On the present map, QTLs were identified for five and six traits in 2008 and 2009, respectively (Table 3 and Fig. 1, thick bars). The phenotypic variations explained by each QTL varied from $9.3 \%$ to $37.7 \%$ (Table 3). A single $\mathrm{OV}$ QTL was detected on Chr I (Fig. 1, pink thick bar). The Saya-S allele resulted in an increase of the $O V$. PS QTLs were detected on $\mathrm{Chr} \mathrm{G}$ in 2008 and on the lower part of $\mathrm{Chr}$ I in 2009 (Fig. 1, green thick bars). On the upper part of Chr I, PS QTL was located within the vicinity in each year. The QTLs on $\mathrm{Chr} \mathrm{G}$ and on the upper part of Chr I had additive effects of the same direction; the Saya-S allele increased the $P S$ scale, whereas the QTL on the lower part of Chr I had an opposite effect (Table 3). Pub QTLs were detected on Chrs $\mathrm{A}$ and $\mathrm{C}$ in both years (Fig. 1, brown thick bars). The QTLs on Chrs A and C explained a proportion of phenotypic variance of $34.4-37.7 \%$ and $24.2-25.3 \%$, respectively and the Haru-S allele resulted in an increase of Pub. QTLs for $W W$ and $U W$ were identified at an overlapped region on $\mathrm{Chr} \mathrm{B}$ in 2009 (Fig. 1, black thick bars). The QTL alleles affecting an increase of $W W$ and $U W$ would come from the Saya-S. $R W$ QTLs were detected on $\mathrm{Chr} \mathrm{H}$ in both years and on $\mathrm{Chr} \mathrm{I}$ in 2009 (Fig. 1, purple thick bars). The Haru-S allele increased $R W$ (Table 3). $M W$ QTLs were found on Chrs $\mathrm{H}$ and I in both years (Fig. 1, blue thick bars). Three of the $M W$ QTLs overlapped with the $R W$ QTLs. These QTLs explained 14.6$33.9 \%$ of phenotypic variation. Like the case in $R W$, the Haru-S allele increased the $M W$. 
Table 2. List of SSR and STS markers developed and modified in this study

\begin{tabular}{|c|c|c|c|c|c|c|}
\hline Marker name ${ }^{a}$ & Marker type & Repeat motif & & Primer sequence $\left(5^{\prime}-3^{\prime}\right)$ & $\begin{array}{l}\text { Product } \\
\text { size }(b p)^{b}\end{array}$ & $\begin{array}{c}\text { GenBank accession } \\
\text { no. }\end{array}$ \\
\hline \multicolumn{7}{|c|}{ Radish genomic SSR markers } \\
\hline \multirow[t]{2}{*}{ REL-4 } & SSR & $(\mathrm{CTT})_{6}$ & Forward & GATACGGAGACAAGAAGAAG & 237 & AB630374 \\
\hline & & & Reverse & CGGACAGAAGAGAAAAAGAC & & \\
\hline \multirow[t]{2}{*}{ REL-12 } & SSR & $(\mathrm{GAA})_{25}$ & Forward & CTTTAACACCAGGAATCC & 330 & AB630375 \\
\hline & & & Reverse & GCCTCTGAAATCCTCCAATC & & \\
\hline \multirow[t]{2}{*}{ REL-13 } & SSR & $(\mathrm{CA})_{13}$ & Forward & CTAGCAATGCATACCAAACAG & 166 & AB630376 \\
\hline & & & Reverse & AACTTGGTCGTTGAGCAG & & \\
\hline \multirow[t]{2}{*}{ REL-16 } & SSR & $(\mathrm{TG})_{13}$ & Forward & ACAGCAACGTTTTCAAGTGCTC & 197 & AB630377 \\
\hline & & & Reverse & CTCACATGCAATGCAATGCATAC & & \\
\hline \multirow[t]{2}{*}{ REL-20 } & SSR & $(\mathrm{TC})_{8}$ & Forward & CСTTCATTCTTTGCTTCTTCC & 162 & AB630378 \\
\hline & & & Reverse & TTGTTTCTTCCCAGAAAGCC & & \\
\hline \multirow[t]{2}{*}{ REL-37 } & SSR & $(\mathrm{GA})_{16}$ & Forward & GAAAACGGGAATCAGATACAAC & 94 & AB630379 \\
\hline & & & Reverse & CTCATTCACTTTCTTCGCCA & & \\
\hline \multirow[t]{2}{*}{ REL-40 } & SSR & $(\mathrm{AC})_{12}$ & Forward & TGATAGCAAAGGCAGAGGTGGC & 176 & AB630380 \\
\hline & & & Reverse & CAAGAAACCTGTCGCAAGGTACAG & & \\
\hline \multirow[t]{2}{*}{ REL-41 } & SSR & $(\mathrm{CA})_{6}$ & Forward & CGTTCATATCAACTTTTTGAGCGAG & 154 & AB630381 \\
\hline & & & Reverse & TTATGCCAGCCTTAGCTGC & & \\
\hline \multicolumn{7}{|c|}{ SSR and STS markers developed from radish ESTs } \\
\hline \multirow[t]{2}{*}{ RES-8 } & SSR & $(\mathrm{T}){ }_{15} \mathrm{GT}$ & Forward & GGATAACAATTACCTCGATCA & 206 & AF248491 \\
\hline & & $\mathrm{C}(\mathrm{A})_{21}$ & Reverse & GGAAACCAAATACCACAAAAG & & \\
\hline \multirow{2}{*}{ RES-10/HaeIII } & STS (CAPS) & - & Forward & CTTCCTAGTAGCTAACTC & 195 & AF263920 \\
\hline & & & Reverse & AACAGTCATGACACGTGG & & \\
\hline \multirow[t]{2}{*}{$\operatorname{RrGGT2/Afa\mathrm {I}}$} & STS (CAPS) & - & Forward & CTATCTCCAGGCACTGGAATCG & 431 & EX761507 \\
\hline & & & Reverse & ATCGTCCCTCCGGCGATTGG & & \\
\hline \multirow{2}{*}{ RsEST-011 } & STS (indel) & - & Forward & ATCATGTCTACGTGCGGAAACT & 353 & AF051121 \\
\hline & & & Reverse & ACCATTACATCACACACCATGC & & \\
\hline \multirow[t]{2}{*}{ RsEST-012 } & STS (indel) & - & Forward & GTACCAAGTTCCCCAGTTATGC & 294 & AF051120 \\
\hline & & & Reverse & GATGCCAACAAAGGACTTGTAA & & \\
\hline \multirow[t]{2}{*}{ RsEST-030/TaqI } & STS (CAPS) & - & Forward & CTATTCCTTTCACCCGTTTCT & 303 & $\mathrm{~T} 25178$ \\
\hline & & & Reverse & CTGACAGACTCTTGCTTCTCAG & & \\
\hline \multirow[t]{2}{*}{ RsEST-038 } & STS (indel) & - & Forward & GATGTATCAGGTGGTGAAAT & 192 & EL738632 \\
\hline & & & Reverse & TACACCACCAGAAAGTCCAC & & \\
\hline RsEST-043/AfaI & STS (CAPS) & - & Forward & ATCATGGTCCGATACACAAATC & 350 & EL738626 \\
\hline & & & Reverse & TGCATTGAAACCAAAAGACC & & \\
\hline RsEST-045/TaqI & STS (CAPS) & - & Forward & GCAGAATTTTCCGCTGTAGTTC & 275 & EL738624 \\
\hline & & & Reverse & TGACAAAATACTTCCTGCAAGC & & \\
\hline$R s F T$ & STS (indel) & - & Forward & TAGGTTTTCTGTTATATCACTGCT & 103 & AB611697 \\
\hline & & & Reverse & AAATCGAGTCTCTGAAAGTTTTAC & & \\
\hline$R s M A F 1$ & STS (indel) & - & Forward & GTCAGGATCCTTGATCGATATGG & 81 & FD958357 \\
\hline & & & Reverse & GCTCAGAGATTTTGACTGAAGAT & & \\
\hline RsSTS1004A/TaqI & STS (CAPS) & - & Forward & ATTTCAATCCACTCAAGCTGAATCT & 522 & AB611698 \\
\hline & & & & TTTTATCTTGCATGGAACTCTAACC & & \\
\hline RsSTS1004B & STS (indel) & - & Forward & TCTAAGCCCATGGTTATGAGAC & 154 & AB611699 \\
\hline & & & Reverse & AAGGAAAGGCCCACTTGAATA & & \\
\hline RsSTS1006B & STS (indel) & - & Forward & CCCTAACGCACAAGTAATTA & 191 & AB611700 \\
\hline & & & Reverse & TAATCCGTGTAGCAGAACAATA & & \\
\hline Rs1SSR7063 & SSR & $(\mathrm{CT})_{7}$ & Forward & GGACAAGTTTATACTTTAAGTG & 223 & 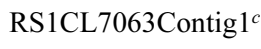 \\
\hline & & & Reverse & GATTTTTGCACAAGTTTTCCTAGAG & & \\
\hline Rs2STS1232/TaqI & STS (CAPS) & - & Forward & AGCCTGGTAAGCATCTCCAA & 468 & RS2CL1232Contig $1^{c}$ \\
\hline & & & Reverse & TGACCATGGTGGGAGTGAAT & & \\
\hline Rs2STS1263/HhaI & STS (CAPS) & - & Forward & GATCCAAGGTTCCATGCCCATG & 366 & RS2CL1263Contig $1^{c}$ \\
\hline & & & Reverse & GACCCGGCAGCATTCTGCTCTA & & \\
\hline Rs2STS2285/AluI & STS (CAPS) & - & Forward & TGTTCTTCAGGGGAGTTTGATT & 489 & RS2CL2285Contig $1^{c}$ \\
\hline & & & Reverse & CCTTGGTCTTCTAGTTGCGTTT & & \\
\hline Rs2STS3325/DdeI & STS (CAPS) & - & Forward & CTAGCTCCAGGGAGTCAATTCA & 517 & RS2CL3325Contig $1^{c}$ \\
\hline & & & Reverse & CCATCTGAGTAACGAGTGCG & & \\
\hline
\end{tabular}


Table 2. (continued)

\begin{tabular}{|c|c|c|c|c|c|c|}
\hline Marker name $^{a}$ & Marker type & Repeat motif & & Primer sequence $\left(5^{\prime}-3^{\prime}\right)$ & $\begin{array}{l}\text { Product } \\
\text { size }(b p)^{b}\end{array}$ & $\begin{array}{c}\text { GenBank accession } \\
\text { no. }\end{array}$ \\
\hline \multirow[t]{2}{*}{ Rs2STS3416 } & STS (indel) & - & Forward & TACCAAAGAAGAGACCCTTATG & 451 & $\mathrm{RS}^{2 C L 3416 C o n t i g 1}{ }^{c}$ \\
\hline & & & Reverse & TCTAAGAACCCTTGATCTTTGT & & \\
\hline \multirow[t]{2}{*}{ Rs2STS6006/HaeIII } & STS (CAPS) & - & Forward & CACAGCCATCACTTGCAGATCC & 539 & RS2CL6006Contig1 $^{c}$ \\
\hline & & & Reverse & ATGCTGCTACTGTACACCGGGT & & \\
\hline \multicolumn{7}{|c|}{ Markers developed from Brassica rapa genomic DNA } \\
\hline \multirow[t]{2}{*}{ BrGL1-like/MspI } & STS (CAPS) & - & Forward & GAACAGATAACCAAGTCAAG & 310 & AC232566 \\
\hline & & & Reverse & TTAAACCAGAAAGCGTTACTGC & & \\
\hline \multicolumn{7}{|c|}{ Marker modified from the previously reported B. rapa SSR } \\
\hline \multirow[t]{2}{*}{ BRMS-036_R/AluI } & STS (CAPS) & - & Forward & GGTCCATTCCTTTTTGCATCTG & 141 & AB630382 \\
\hline & & & Reverse & GGGTAACAAACATTTCTTAACCTCTC & & \\
\hline \multirow[t]{2}{*}{ BRMS-231_R } & STS (indel) & - & Forward & CGGTGTATTTCCATTTATGCGTTT & 180 & CU695294 \\
\hline & & & Reverse & TTCTGGACTTCAATTAGAGATGGTC & & \\
\hline \multicolumn{7}{|c|}{ Markers developed from Arabidopsis genome data } \\
\hline \multirow[t]{2}{*}{ AtSTS-64 } & STS (indel) & - & Forward & CTCTCGGGTAGTTTACCTGACTTC & 640 & At3g05990d \\
\hline & & & Reverse & GAACTTGGCCAGTTAGGTTATTGT & & \\
\hline \multirow[t]{2}{*}{ AtSTS-1015 } & STS (indel) & - & Forward & GTTGGTTAGGCTTAAACAGATG & 850 & At1g74860 $d$ \\
\hline & & & Reverse & TCСТАСАATATCTTCAGCTTCTC & & \\
\hline \multirow[t]{2}{*}{ AtSTS-1027 } & STS (indel) & - & Forward & TAAAACCTATCATCTCCGTACC & 880 & At1g $71950^{d}$ \\
\hline & & & Reverse & GCACAACTTGAATCACACC & & \\
\hline
\end{tabular}

${ }^{a}$ In CAPS markers, restriction enzymes used for polymorphism detection are indicated after slashes (/).

${ }^{b}$ Sizes are estimated by agarose gel electrophoresis or predicted from the sequences used for primer design.

${ }^{c}$ For markers designed from radish cDNA contig data, the cDNA contig nos. are shown instead of GenBank accession nos. (their sequences are shown in Supplemental Fig. 3).

${ }^{d}$ For markers designed from Arabidopsis genome data, the locus tags are shown instead of GenBank accession nos.

Table 3. Map positions and effect of QTLs detected in RILs

\begin{tabular}{|c|c|c|c|c|c|c|c|}
\hline Trait & Year & Chr & Map position in cM (peak) ${ }^{a}$ & Marker interval $^{b}$ & $\mathrm{LOD}^{c}$ & $R^{2}(\%)^{d}$ & Additive effect ${ }^{c}$ \\
\hline$O V$ & 2008 & I & $10.9-14.9(11.9)$ & RES-11-E6M5_148 & 3.5 & 11.8 & 0.79 \\
\hline \multirow[t]{4}{*}{$P S$} & 2008 & G & $42.0-50.7(45.0)$ & RsHH019-E6M4_87 & 3.8 & 10.8 & 0.31 \\
\hline & & I & $8.5-8.9(8.5)$ & RM_42-RES-11 & 6.4 & 20.9 & 0.40 \\
\hline & 2009 & I & $2.6-3.0(2.6)$ & RM_16-E6M5_99 & 3.5 & 16.9 & 0.32 \\
\hline & & I & $67.4-75.9(68.6)$ & E2M4_196-RsSR094 & 3.2 & 11.9 & -0.27 \\
\hline \multirow[t]{4}{*}{$P u b$} & 2008 & A & $72.2-78.1(77.1)$ & RsSR104-RsSA083 & 13.6 & 37.7 & -0.86 \\
\hline & & $\mathrm{C}$ & $42.6-46.1(44.1)$ & E1M7_238-Rs2STS1232/TaqI & 9.7 & 24.2 & -0.68 \\
\hline & 2009 & A & $72.2-78.1(77.1)$ & RsSR104-RsSA083 & 12.8 & 34.4 & -0.82 \\
\hline & & $\mathrm{C}$ & $36.1-38.3(38.3)$ & RM_1-E5M8_427 & 10.1 & 25.3 & -0.69 \\
\hline$W W$ & 2009 & B & $1.8-2.8(2.8)$ & AtSTS-1015-REL-4 & 3.8 & 18.7 & 213.34 \\
\hline$U W$ & 2009 & B & $1.8-2.8(2.8)$ & AtSTS-1015-REL-4 & 3.6 & 17.8 & 175.33 \\
\hline \multirow[t]{3}{*}{$R W$} & 2008 & $\mathrm{H}$ & $34.7-35.6(35.6)$ & RsHH012-E3M3_77 & 3.7 & 9.3 & -51.95 \\
\hline & 2009 & $\mathrm{H}$ & $24.9-25.7(24.9)$ & RES-1-SLG_CAPS/PleI & 11.6 & 33.9 & -113.01 \\
\hline & & I & $48.5-51.9(48.9)$ & REL-13-RES-5/HapII & 6.6 & 16.8 & -82.24 \\
\hline \multirow[t]{4}{*}{$M W$} & 2008 & $\mathrm{H}$ & $32.7-36.6(35.6)$ & RsHH012-E3M2_105 & 8.4 & 25.1 & -53.52 \\
\hline & & I & $42.2-48.5(46.2)$ & E5M4_90-REL-13 & 5.4 & 14.6 & -42.84 \\
\hline & 2009 & $\mathrm{H}$ & $24.9-25.7(24.9)$ & RES-1-SLG_CAPS/PleI & 11.7 & 33.9 & -107.27 \\
\hline & & I & $48.5-51.9(48.9)$ & REL-13-RES-5/HapII & 6.9 & 17.3 & -79.06 \\
\hline
\end{tabular}

${ }^{a}$ Region above the LOD threshold, in which the peak position is in parentheses.

${ }^{b}$ Smallest marker interval flanking a region above the LOD threshold.

${ }^{c}$ Scores at the LOD peaks.

${ }^{d}$ Phenotypic variation explained by each QTL at the LOD peak. 


\section{Discussion}

Recently, we made radish linkage maps using $F_{1}$ and $F_{2}$ populations (Budahn et al. 2009, Kamei et al. 2010, Nakatsuji et al. 2011, Tsuro et al. 2005, 2008). These studies included limited number of specific markers derived from radish. Here we located 134 radish-specific markers (97 SSRs and 37 STSs) on the map using RILs. The map consisted of nine LGs, which corresponds to the haploid chromosome number of radish. Based on the anchor markers, correspondence of LGs between this study and $\mathrm{Li}$ et al. (2011) has been accomplished (Fig. 1, asterisks and parentheses). Through these maps, LGs from different studies (Kamei et al. 2010, Nakatsuji et al. 2011, Shirasawa et al. 2011) can also be assigned (data not shown). The total length of our map $(673.6 \mathrm{cM})$ was shorter than those of $\mathrm{Li}$ et al. (2011) and Shirasawa et al. (2011). Assumed from the positions of anchor markers and the distributions of syntenic regions, the present map was roughly estimated to cover $>70 \%$ of the above two maps (data not shown). Since our map still includes anonymous AFLPs and STSs that did not hit to Arabidopsis locus, the value might be underestimated. Thus, our map is considered to cover a significant fraction of the radish genome.

Two recent reports (Li et al. 2011, Shirasawa et al. 2011) involved many more markers and gave a more detailed picture of Arabidopsis synteny than this study. However, the present map has the following two advantages over the previous maps. First, each LG on the present map was assigned to a radish chromosome (Fig. 1, gray boxes). Previous work of chromosome-LG assignment was done with anonymous RAPD markers (Budahn et al. 2009). In this study, the assignment has been completed with radishspecific markers (Supplemental Fig. 5). This allows us to conduct easier and more concrete chromosome-LG assignment than in many other radish maps. Although Akaba et al. (2009) have made monosomic rape-radish addition lines, no assignment to radish linkage map has been reported. The chromosomal correspondence to the set of addition lines in Akaba et al. (2009) and ours should be considered in the future. Second, by using the present map, several QTLs for morphological traits were detected. Some of the QTLs showed low consistency between years probably because of an environmental effect. Indeed, the weight traits in 2008 were mostly smaller than those in 2009 (Supplemental Fig. 4E-4H). Low precipitation during the primary growth of radish could be a reason for such difference, because the monthly total precipitation in October, 2008 was lower than that of the climate normal (averages of 1997-2005) (data not shown). Unfortunately, the seeds of RILs were insufficient to assess the possibility of environmental effects and genotype-environment interaction by planting several fields. In contrast to the QTLs with low consistency, QTLs that were reproducible in the two years suggest the presence of loci with seemingly stable effects. The markers linked to the stable QTLs could provide practical tools for selecting rad- ish lines. The details of QTLs are further described below.

The ovule and seed number traits in our radish population were quantitative. Although the $S E$ remains to score in the rat-tailed radish inbred line Saya-S, presumably the Saya-S had more $S E$ than the Haru-S, assumed from a report describing that seed numbers in rat-tailed radishes were $\geq 8$ (Yazawa et al. 1953). In Arabidopsis, both of the $O V$ and $S E$ traits were also quantitative, in which QTLs overlapped on chrs 1 and 2 (Alonso-Blanco et al. 1999). In wild radish R. raphanistrum, the ovule number had a positive effect on the seed number (Conner et al. 1996). Unlike these cases, in this study, only an $O V$ QTL has been found whereas no $S E$ QTL has been detected. Such discrepancy might be because the number of ovules becoming seeds may be influenced by factors such as the ovule number itself, the quantity and quality of pollen transferred, nutrients and photosynthate (Lee 1988).

Two QTLs for pubescence were found in radish. There is no report of a pubescence locus on a radish map, whereas many reports of B. rapa studies (Ågren and Schemske 1992, Kubo et al. 2010, Li et al. 2009, Lou et al. 2007, Nozaki et al. 1997, Song et al. 1995, Teutonico and Osborn 1994, Zhang et al. 2009) have indicated that the pubescence is controlled by a single locus with the proposal of a few candidate genes. In the present case, a marker made from the B. rapa candidate gene ( $B r G L 1)$ was mapped at the peak position of the Pub QTL on Chr A (Table 3 and Fig. 1). This gene is a good candidate for a $P u b$ gene in our population; however, we have no information regarding the second $P u b$ gene in the radish. The $P u b$ QTLs found in this study are potentially used for breeding a pest-resistant radish line, as utilized in Brassica crops (Ågren and Schemske 1992).

The plant shape (e.g. leaf angle) is an important trait for cultivation because it largely affects planting density and effectiveness of receiving solar energy. This trait has extensively been studied in maize and rice (Tian et al. 2011 and references therein), whereas little is known for Brassicaceae crops. In the PS QTLs identified here, the rat-tail radish allele would influence the plant shape into an erect form (Table 3). Although this trait might be environmentally variable, these QTLs could be useful for the selection of radish lines with a more compact plant shape, thus improving canopy.

Root formation is one of the most important traits for the Asian radish cultivars, because a thick and long cylindrical root is indispensable for Asian cultivars. In the present population between rat-tail and Japanese radishes (Saya-S and Haru-S), most $M W$ QTLs were associated with $R W$ QTLs (Table 3 and Fig. 1). This clearly demonstrated that thickness of the main root contributed to the total root weight. In this study, we have found two potential QTL clusters for root formation. We previously identified four genomic regions of root-related QTLs using an $\mathrm{F}_{2}$ population between Chinese and Japanese radishes (Tsuro et al. 2008). No significant overlap was detectable in the positions of root QTLs between the previous and the present studies (data not 
shown). The root formation QTL might act in a cultivarspecific manner.

In conclusion, we have constructed a chromosomeassigned, sequence-tagged linkage map of the radish, and have identified several QTLs for morphological traits. The present data will provide useful information for further genetic studies and for selection during radish breeding programs. As an example, the QTL detected in radish could be introduced into Brassica crops via homoeologous recombination as in the case of the fertility restorer gene (Sakai et al. 1996). Genetic markers closely linked to the loci are powerful tools for detecting recombination in interspecific hybrids. For this purpose, the present chromosome-assigned, sequence-tagged linkage map of the radish would be useful for MAS and chromosome manipulation not only in the radish, but also in a wide range of Brassica crops.

\section{Acknowledgments}

We are grateful to Prof. T. Nishio and Dr. S. Matsumoto for providing the marker information. We thank Dr. N. Wang, Mr. M. Saito and Mr. A. Kamei for development of part of the markers and Ms. H. Kasaoka, Ms. A. Garve, Ms. K. Maier and Ms. E. Koblitz for technical assistance. This work was partly supported by the Program for Promotion of Basic and Applied Researches for Innovations in Bio-oriented Industry (BRAIN) to M.H. and JSPS Kakenhi 23510236 to N.K.

\section{Literature Cited}

Ågren,J. and D.W.Schemske (1992) Artificial selection on trichome number in Brassica rapa. Theor. Appl. Genet. 83: 673-678.

Akaba,M., Y.Kaneko, Y.Ito, Y.Nakata, S.W.Bang and Y.Matsuzawa (2009) Production and characterization of Brassica napusRaphanus sativus monosomic addition lines mediated by the synthetic amphidiploid "Raphanobrassica". Breed. Sci. 59: 109118.

Alonso-Blanco, C., H. Blankestijn-de Vries, C.J. Hanhart and M. Koornneef (1999) Natural allelic variation at seed size loci in relation to other life history traits of Arabidopsis thaliana. Proc. Natl. Acad. Sci. USA 96: 4710-4717.

Bett, K.E. and D.J.Lydiate (2003) Genetic analysis and genome mapping in Raphanus. Genome 46: 423-430.

Budahn,H., O.Schrader and H.Peterka (2008) Development of a complete set of disomic rape-radish chromosome-addition lines. Euphytica 162: 117-128.

Budahn,H., H.Peterka, M.A.A.Mousa, Y.Ding, S.Zhang and J.Li (2009) Molecular mapping in oil radish (Raphanus sativus L.) and QTL analysis of resistance against beet cyst nematode (Heterodera schachtii). Theor. Appl. Genet. 118: 775-782.

Conner,J.K., S.Rush and P.Jennetten (1996) Measurements of natural selection on floral traits in wild radish (Raphanus raphanistrum). I. Selection through lifetime female fitness. Evolution 50: 11271136 .

Crisp,P. (1995) Radish, Raphanus sativus (Cruciferae). In: Smartt,J. and N.W.Simmonds (eds.) Evolution of Crop Plants, 2nd edn. Longman Scientific \& Technical, Harlow, pp. 86-89.
Furusato,K. and A.Miyazawa (1958) Japanese radish cultivars considered viewed from horticulture. In: Nishiyama,I. (ed.) Japanese Radish, Japan Society for the Promotion of Science, Tokyo, pp. $138-161$.

Jones, N., H.Ougham, H.Thomas and I.Pašakinskienè (2009) Markers and mapping revisited: finding your gene. New Phytol. 183: 935966.

Kamei,A., M.Tsuro, N.Kubo, T.Hayashi, N.Wang, T.Fujimura and M.Hirai (2010) QTL mapping of clubroot resistance in radish (Raphanus sativus L.). Theor. Appl. Genet. 120: 1021-1027.

Kaneko,Y., C.Kimizuka-Takagi, S.W.Bang and Y.Matsuzawa (2007) Radish. In: Kole, C. (ed.) Genome Mapping and Molecular Breeding in Plants, vol. 5 Vegetables, Springer-Verlag, Heidelberg, pp. 141-160.

Kitamura, S. (1958) Cultivars of radish and their change. In: Nishiyama,I. (ed.) Japanese Radish, Japan Society for the Promotion of Science, Tokyo, pp. 1-19.

Kubo,N., M.Saito, H.Tsukazaki, T.Kondo, S.Matsumoto and M.Hirai (2010) Detection of quantitative trait loci controlling morphological traits in Brassica rapa L. Breed. Sci. 60: 164-171.

Lee,T.D. (1988) Patterns of fruit and seed production. In: Doust,J.L. and L.L.Doust (eds.) Plant Reproductive Ecology: Patterns and Strategies, Oxford University Press, New York, pp. 179-202.

Li,F., H.Kitashiba, K.Inaba and T.Nishio (2009) A Brassica rapa linkage map of EST-based SNP markers for identification of candidate genes controlling flowering time and leaf morphological traits. DNA Res. 16: 311-323.

Li,F., Y.Hasegawa, M.Saito, S.Shirasawa, A.Fukushima, T.Ito, H. Fujii, S.Kishitani, H.Kitashiba and T.Nishio (2011) Extensive chromosome homoeology among Brassiceae species were revealed by comparative genetic mapping with high-density EST-based SNP markers in radish (Raphanus sativus L.). DNA Res. 18: 401411 .

Lou,P., J.Zhao, J.S.Kim, S.Shen, D.P.Del Carpio, X.Song, M.Jin, D.Vreugdenhil, X.Wang, M.Koornneef et al. (2007) Quantitative trait loci for flowering time and morphological traits in multiple populations of Brassica rapa. J. Exp. Bot. 58: 4005-4016.

Nakatsuji, R., T.Hashida, N.Matsumoto, M.Tsuro, N.Kubo and M. Hirai (2011) Development of genomic and EST-SSR markers in radish (Raphanus sativus L.). Breed. Sci. 61: 413-419.

Niikura,S. and S.Matsuura (1998) Identification of self-incompatibility alleles $(S)$ by PCR-RFLP in radish (Raphanus sativus L.). Euphytica 102: 379-384.

Nozaki,T., A.Kumazaki, T.Koba, K.Ishikawa and H.Ikehashi (1997) Linkage analysis among loci for RAPDs, isozymes and some agronomic traits in Brassica campestris L. Euphytica 95: 115-123.

Peterka,H., H.Budahn, O.Schrader, R.Ahne and W.Schütze (2004) Transfer of resistance against the beet cyst nematode from radish (Raphanus sativus) to rape (Brassica napus) by monosomic chromosome addition. Theor. Appl. Genet. 109: 30-41.

Saito,M., N.Kubo, S.Matsumoto, K.Suwabe, M.Tsukada and M.Hirai (2006) Fine mapping of the clubroot resistance gene, Crr3, in Brassica rapa. Theor. Appl. Genet. 114: 81-91.

Sakai,T., H.J.Liu, M.Iwabuchi and J.Kohno-Murase (1996) Introduction of a gene from fertility restored radish (Raphanus sativus) into Brassica napus by fusion of $\mathrm{X}$-irradiated protoplasts from a radish restorer line and iodacetoamide-treated protoplasts from a cytoplasmic male-sterile cybrid of B. napus. Theor. Appl. Genet. 93: 373-379.

Schmidt,R. and I.Bancroft (2011) Plant genetics and genomics: crops and models, vol. 9, Genetics and genomics of the Brassicaceae. 
Springer Science and Business Media, New York.

Shirasawa,K., M.Oyama, H.Hirakawa, S.Sato, S.Tabata, T.Fujioka, C.Kimizuka-Takagi, S.Sasamoto, A.Watanabe, M. Kato et al. (2011) An EST-SSR linkage map of Raphanus sativus and comparative genomics of the Brassicaceae. DNA Res. 18: 221-232.

Song,K., M.K.Slocum and T.C.Osborn (1995) Molecular marker analysis of genes controlling morphological variation in Brassica rapa (syn. campestris). Theor. Appl. Genet. 90: 1-10.

Suwabe, K., H.Tsukazaki, H.Iketani, K.Hatakeyama, M.Kondo, M. Fujimura, T.Nunome, H.Fukuoka, M. Hirai and S.Matsumoto (2006) Simple sequence repeat-based comparative genomics between Brassica rapa and Arabidopsis thaliana: the genetic origin of clubroot resistance. Genetics 173: 309-319.

Teutonico,R.A. and T.C.Osborn (1994) Mapping of RFLP and qualitative trait loci in Brassica rapa and comparison to the linkage maps of B. napus, B. oleracea, and Arabidopsis thaliana. Theor. Appl. Genet. 89: 885-894.

Tian,F., P.J.Bradbury, P.J.Brown, H.Hung, Q.Sun, S.Flint-Garcia, T.R.Rocheford, M.D.McMullen, J.B.Holland and E.S.Buckler (2011) Genome-wide association study of leaf architecture in the maize nested association mapping population. Nat. Genet. 43: 159162.

Tsuro, M., K.Suwabe, N.Kubo, S.Matsumoto and M.Hirai (2005) Construction of a molecular linkage map of radish (Raphanus sativus L.), based on AFLP and Brassica-SSR markers. Breed. Sci. 55: $107-111$

Tsuro, M., K.Suwabe, N.Kubo, S.Matsumoto and M.Hirai (2008)
Mapping of QTLs controlling for root shape and red pigmentation in radish, Raphanus sativus L. Breed. Sci. 58: 55-61.

Van Ooijen,J.W. (2009) MapQTL 6, software for the mapping of quantitative trait loci in experimental populations of diploid species. Kyazma BV, Wageningen.

Van Ooijen,J.W. and R.E.Voorrips (2001) JoinMap Version 3.0, Software for the calculation of genetic linkage maps. Plant Research International, Wageningen.

Wang,N., J.Hu, R.Ohsawa, M.Ohta and T.Fujimura (2007) Identification and characterization of microsatellite markers derived from expressed sequence tags (ESTs) of radish (Raphanus sativus L.). Mol. Ecol. Notes 7: 503-506.

Wang,X., H.Wang, J.Wang, R.Sun, J.Wu, S.Liu, Y.Bai, J.H.Mun, I. Bancroft, F.Cheng et al. (2011) The genome of the mesopolyploid crop species Brassica rapa. Nat. Genet. 43: 1035-1039.

Xu,L., L.Wang, Y.Gong, W.Dai, Y.Wang, X.Zhu, T.Wen and L.Liu (2012) Genetic linkage map construction and QTL mapping of cadmium accumulation in radish (Raphanus sativus L.). Theor. Appl. Genet. 125: 659-670.

Yazawa,S., T.Asahira, Y.Matsumoto and T.Namiki (1953) Morphological and physiological characteristics of rat-tailed radish introduced from India and Thailand. Agriculture and Horticulture 58: 704-706.

Zhang,J., Y.Lu, Y.Yuan, X.Zhang, J.Geng, Y.Chen, S.Cloutier, P.B.E.McVetty and G.Li (2009) Map-based cloning and characterization of a gene controlling hairiness and seed coat color traits in Brassica rapa. Plant Mol. Biol. 69: 553-563. 